\title{
Integrating Simulation Scenarios and Clinical Practices Guided by Concepts of Translational Medicine
}

\author{
Jing Yang ${ }^{1}$, Si-min Huang ${ }^{1}, \mathrm{Ze}$-jian $\mathrm{Li}^{2}, \mathrm{Lie} \mathrm{Feng}^{1}$ \& Chun-ting $\mathrm{Lu}^{3}$ \\ ${ }^{1}$ Department of Internal Medicine, First Affiliated Hospital of Jinan University, Guangzhou, China \\ ${ }^{2}$ Medical Centre of Stomatology, First Affiliated Hospital of Jinan University, Guangzhou, China \\ ${ }^{3}$ The Science \& Education Office, First Affiliated Hospital, Jinan University, Guangzhou, China \\ Correspondence: Chun-ting Lu, The Science \& Education Office, First Affiliated Hospital, Jinan University, \\ Guangzhou, 510630, China
}

Received: November 22, 2017

Accepted: December 19, 2017

Online Published: January 4, 2018

doi:10.5430/ijhe.v7n1p10

URL: https://doi.org/10.5430/ijhe.v7n1p10

\section{Funding Information:}

This work was supported by Teaching Research Programs of Higher Medical Education of Ministry of Education in 2016 (grant number: Edu-CR-2016-4-2, Edu-CR-2016-4-3 and Edu-CR-2016-6-2), Education Teaching Reform Project of Guangdong Province (grant number: 85514022), Education and Teaching Reform Project of Jinan University (grant number: JG2015116 and JG2016028) and Fundamental Research for the Central Universities (grant number: 216174100)

\begin{abstract}
Purpose: To develop a novel method for closely and effectively integrating simulation scenarios and clinical practices to improve clinical skills training in the concepts of translational medicine.

Methods: Forty-two and 38 third-year medical students in the classes of 2010 and 2009 at Jinan University were selected as an observation group and a control group, respectively. The former group was taught according to a new, integrated mode, while the latter received traditional methods. Students' scores on practical tests in physical examination, internal punctures, and case analysis; theory-based exams on diagnostics and internal medicine; and questionnaire surveys were compared and analyzed. In addition, system-oriented curricula were explored and implemented.

Results: A novel mode that closely and effectively integrates theory and practice in the observation group had been established although there were no statistically significant difference $(P>0.05)$ between Grade 2010 and Grade 2009 in clinical basic skills training scores. However, there were statistically significant differences $(P<0.05)$ in scores on practical tests of physical examination and internal punctures among the diagnostic, internal medicine and internship periods in the class of 2010 but no statistically significant difference $(P>0.05)$ in case analysis scores. Therefore, system-oriented curricula were initially designed and explored in excellent students from Grade 2010 to reinforce clinical thinking.

Conclusion: The novel program integrating simulation scenarios and clinical situations for training students in diagnostics and internal medicine skills can improve medical students' clinical comprehensive abilities and achieve effects that are similar to those of the traditional method. This program is more popular with students and ensures patient safety as well. In addition, different characteristics of clinical skills training have been compared for the further exporation of system-oriented curricula.
\end{abstract}

Keywords: Simulation scenarios, Medical simulation, Translational medicine, Patient safety, Clinical basic skills

\section{Introduction}

Current medical education trends emphasize the combination of medical teaching and clinical application to enable students to solve basic and clinical problems effectively. Clinical medicine education is typically given in isolated units, separating theoretical lectures, clinical skills training and clinical bedside teaching rather than integrating them. This teaching paradigm may hinder medical students' ability to apply the knowledge and skills learned in the 
classroom, laboratory or simulation center to the management of real-world clinical problems. Students usually lack guidance from bench to bedside; in particular, there is generally no effective integration of basic knowledge and clinical practice. Moreover, there are insufficient opportunities for students to immediately practice diagnosis and treatment procedures in clinical cases. In view of this situation, a method for training students using highly and flexibly integrated clinical skills in the medical simulation center and teaching hospital was developed by drawing inspiration from the concept of translational medicine (TM) which has been providing a good idea to avoid the isolation of classroom or laboratory teaching from hospital or bedside teaching.

TM is an up-to-date discipline which was first originated from biomedical science enabled bench to bedside approaches between diagnostic and therapeutic measures effectively (Woolf, 2008). It belongs to the interdisciplinary field in order to make the resources shared better in health care system involved in different aspects (Cohrs, Martin, Ghahramani, Bidaut, \& J, 2015). TM as an advanced subject has gained increasing attention since its establishment in the international medical field. Its main purpose is to closely connect basic medical science and clinical situations(Yuan, 2014). The core of TM research is to establish an effective connection between researchers who are engaged in basic scientific discovery and doctors who understand the needs of patients. Considerable efforts have been made to shorten the progression from basic research to clinical application(Dai, Yang, \& Gan., 2013). Simulation is considered a technique, not a technology, and relies heavily on the instructional design(Gaba, 2007). Simulation can be integrated naturally with other educational methodologies. There is a need to integrate different disciplinary perspectives and inter-professional learning opportunities(Moral \& Maestre., 2013). Accordingly, the fact that most clinical skills training in early medical education is isolated or concentrated in the laboratory or simulation center, separate from the hospital, can be addressed. Learning objectives can be adjusted through an iterative process based on realistic limitations of scheduling, the balance among various learning modalities within the module, the feasibility of standardized patient (SP) scenario design, and faculty availability(Miyasaka, Buchholz, LaMarra, Karakousis, \& Aggarwal, 2015). TM acts as a bridge for the bi-directional conversion between basic research and clinical practice(Xi-ming \& Jun-hon, 2014). Furthermore, for teaching hospitals, there are unprecedented challenges in both ensuring the teaching quality while not exacerbating the patients' conditions. Therefore, it is necessary to provide undergraduate patient safety education and establish the concept of "patient safety" in every area of medical services(Ming-ming, Yu-rong, \& Jing, 2010). Many courses use simulation, such as in patient safety and the practice of skills in emergency conditions(W. Bond et al., 2008; W. F. Bond et al., 2007). Simulation has many benefits for students, including providing them with realistic experiences while causing no harm to patients; moreover, it is a student-centered learning style(Vattanavanit, Kawla-Ied, \& Bhurayanontachai, 2017). The combination of simulation training and clinical practice has greatly remedied the shortcomings of merely teaching theory without providing the opportunity for students to practice with real patients(Back et al., 2014). Therefore, it is also important to focus on fostering medical students' capacity to take good care of the patient by cautiously considering and avoiding harmful practices during the education process.

\section{Method}

\subsection{Participants and Setting}

Forty-two students were selected from the 5-year clinical medicine major in the class of 2010 as the observation group, while 38 students in the class of 2009 were selected as the control group at Jinan University. Both group teaching tasks were responsible by our department. The observation group in the third year was instructed using the combination mode incorporating medical or virtual simulation technology and clinical practice. In contrast, the control group, also in the third year, was taught using traditional clinical teaching approaches, in which aspects including theoretical lectures, simulation training, and bedside teaching were separated. Both lecture and practical teaching hours were identical in the two groups. The teachers responsible for the two groups' arrangement and teaching materials were also the same. The faculty team had extensive clinical and teaching experience and could ensure smooth conduction of the research.

Throughout the process of teaching and learning, a reasonable program was explored and studied to increase the medical students' comprehensive ability. Each part of the program was arranged as shown in Table 1. The above course content was finished in three semesters, proceeding successively through the diagnostic period, internal medicine period, and internship period. 


\subsection{Teaching Methods}

\subsubsection{Observation Group}

This group was taught using an interactive and integrated mode incorporating simulation scenarios via medical or virtual technology and clinical practice. Simulation scenarios were combined and alternated with bedside teaching. This method not only encourages the students' enthusiasm to learn but also trains them in clinical thinking and capacities adapted to real-world diagnostic and therapeutic problems in the hospital. During the research, several items were chosen as observation projects: physical examination and theory-based examination of diagnostics and internal medicine occurred after course study, while internal punctures and case analysis in the final exam occurred after internship rotation in the ward.

\subsubsection{Control Group}

This group was instructed using the traditional mode. Initially, lectures were taught in the classroom. After theoretical lectures were completed, the students met in the clinical skills training center for concentrated practice in the clinical techniques, and they were trained on the medical simulator repeatedly to develop their skills before they could practice on real patients. Finally, the students were prepared to learn how to practice in the hospital to strengthen and verify the previous clinical concepts and analysis.

\subsubsection{Evaluation of Teaching and Student Performance Outcomes}

1) Practical tests of physical examination (PE), internal punctures, and case analysis were conducted in both groups. The faculty responsible for the evaluation was the same.

2) Theory-based exams of diagnostics and internal medicine.

3) Questionnaire survey to receive feedback from the two groups.

The questionnaire contents mainly reflected students' satisfaction and suggestions regarding the new medical teaching mode. After the completion of theory-based and practical teaching, the 80 students in the two groups were asked to fill in the 'Feedback Opinion Form for Teaching and Learning of Clinical Basic Skills.' Forty-two questionnaires in the observation group and 38 in the control group were sent out, and 40 and 37 were returned, respectively, producing return ratios of $95 \%$ and $97 \%$.

\subsection{Statistical Analysis}

Statistical analyses were performed using the Statistical Package for Social Sciences, version 16.0 (SPSS). Data were recorded in Excel tables, then statistically analyzed using SPSS 16.0. The results are described as the mean \pm standard deviation $\left(X_{ \pm \mathrm{SD}}\right)$. The scores of the two groups were compared, including PE, internal punctures, diagnostics, internal medicine, and case analysis. $P<0.05$ was considered as a statistically significant difference.

\section{Results}

\subsection{A novel Mode that Closely and Effectively Integrates Theory and Practice in the Observation Group}

3.1.1 Teaching PE and Internal Punctures Based on the Combination of Simulation Scenarios and Real Clinical Situations

When visiting the patients in the hospital, the students were first instructed to inquire about their medical history and make a mental list of physical examinations. Then, they were to perform those less harmful and more cooperative PE items on the real patient in the ward to obtain more clinical experience and doctor-patient communication skills, such as inspection and auscultation of the patient. However, for those examinations that the patients may reject the demonstration or performance of, such as palpation or percussion of the lung, the heart, or abdomen, the students were directed to the clinical skills comprehensive training center to practice those items on the medical or virtual simulation teaching system for cardio-pulmonary and abdominal examination.

For example, one patient was suspected and subsequently diagnosed with chronic myelogenous leukemia (CML) with serious splenomegaly, and it was impossible for all students to palpate the spleen on this patient. In this case, only one or two student representatives had the chance to perform the physical examination after taking the patient's history, and most of them needed to return to the medical simulation training center for more practice combined with realistic scenarios. Moreover, diagnostic and therapeutic techniques such as bone marrow aspiration were practiced on the simulator. The results of bone marrow investigation using the graphic analysis system in the clinical skills training center were provided immediately after contact with the real cases. This approach was helpful for students to combine theoretical knowledge and clinically relevant conditions and to avoid discrepancy between the laboratory or simulation center and clinical teaching. When the students enter the internship rotation, they can master their 
examination skills and improve their clinical decision-making in real cases.

Therefore, the students in this group can learn and practice the diagnostic and management process in a specific way that can help them to learn more clinical skills and ensure patient safety at the same time based on the real patient's background.

\subsection{The Arrangement of Each Part among Diagnostics and Internal Medicine Courses.}

During the whole process of teaching and learning, reasonable program was explored and studied in order to increase comprehensive ability of the medical students. Each parts were showed inTable1.

3.2.1 Lectures were taught in the classroom.

3.2.2 The students should be trained on the medical simulator repeatedly and skillfully before they can practice on the real patient.

3.2.3 The students were ready to learn how to practice on the real patient to strengthen and verify the previous clinical idea and analysis.

3.2.4 Multidisciplinary integration of clinical information and basic medical science, and clinical skills based on the cases in the ward or the scenarios.

Table 1. Clinical Skills Training Program in two groups

\begin{tabular}{llllc}
\hline \multicolumn{1}{c}{\begin{tabular}{c} 
Teaching section/Teaching hours \\
\multicolumn{1}{c}{ / Internship weeks }
\end{tabular}} & Lecture & $\begin{array}{c}\text { Simulation-oriented } \\
\text { Training }\end{array}$ & Bedside Practice & $\begin{array}{l}\text { Integration of } \\
\text { Case Study }\end{array}$ \\
\hline PE & 46 & 36 & 18 & 18 \\
Four Internal Punctures & 8 & 8 & 8 & 8 \\
Diagnostics & 72 & 16 & 20 & 18 \\
Internal Medicine & 72 & 8 & 64 & 132 \\
Internship of Internal Medicine & - & - & 128 & 128 \\
\hline
\end{tabular}

PE: Physical examination

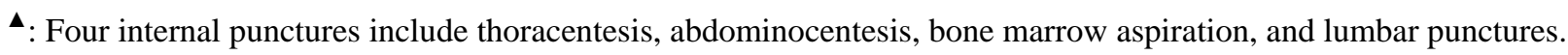

3.3 Comparison of the Mean Scores on Clinical Basic Skills Tests of Diagnostics and Internal Medicine between the Two Groups

As illustrated in Table 2, there were no statistically significant differences $(P>0.05)$ between the observation group and the control group in practical tests of PE, internal punctures and case analysis or in theory-based examinations of diagnostics and internal medicine.

Table 2. Comparison of the Mean Scores of Clinical Basic Skills Tests of Diagnostics and Internal medicine between Two Groups

\begin{tabular}{lcccc}
\hline Indices & Observation group\# & Control group\# & $\mathrm{t}$ & $P$ \\
\hline PE & $86.24 \pm 8.86$ & $84.92 \pm 6.34$ & 0.77 & 0.451 \\
Theory examination of diagnostics & $67.54 \pm 12.21$ & $67.55 \pm 11.88$ & 0.01 & 0.996 \\
Theory examination of internal medicine & $72.61 \pm 9.72$ & $75.10 \pm 8.28$ & 1.22 & 0.227 \\
Internal punctures & $87.13 \pm 9.47$ & $82.78 \pm 9.90$ & 1.96 & 0.054 \\
Case analysis & $75.95 \pm 16.73$ & $81.00 \pm 19.22$ & 1.22 & 0.225 \\
\hline
\end{tabular}

PE: Physical examination

\#: expressed as mean $\pm \mathrm{SD}$

3.4 Comparison of the Mean Scores on PE and Internal Punctures Tests among the Diagnostic Period, Internal Medicine Period, and Internship Period in the Class of 2010.

As illustrated in Table 3 and Fig. 1, data conformed to a normal distribution and are expressed as $\bar{X}_{ \pm \mathrm{SD}}$. Two-tailed $p$-values $<0.05$ were considered statistically significant. 
Table 3. Comparison of the Mean Scores of PE and Internal Punctures Tests among Diagnostic

Period, Internal Medicine Period, Internship Period in Grade 2010.

\begin{tabular}{lllllc}
\hline Indices & Diagnostic Period $^{\#}$ & Internal Medicine Period $^{\#}$ & Internship Period $^{\#}$ & $\mathrm{~F}$ & $P$ \\
\hline $\mathrm{PE}$ & $86.24 \pm 8.87$ & $78.15 \pm 9.68$ & $81.95 \pm 11.24$ & 9.20 & $0.000^{* *}$ \\
Internal punctures & $83.23 \pm 10.37$ & $72.26 \pm 10.27$ & $87.13 \pm 9.47$ & 27.07 & $0.000^{* *}$ \\
\hline
\end{tabular}

PE: Physical examination

**: $P<0.01$ between two groups; \#expressed as mean \pm SD

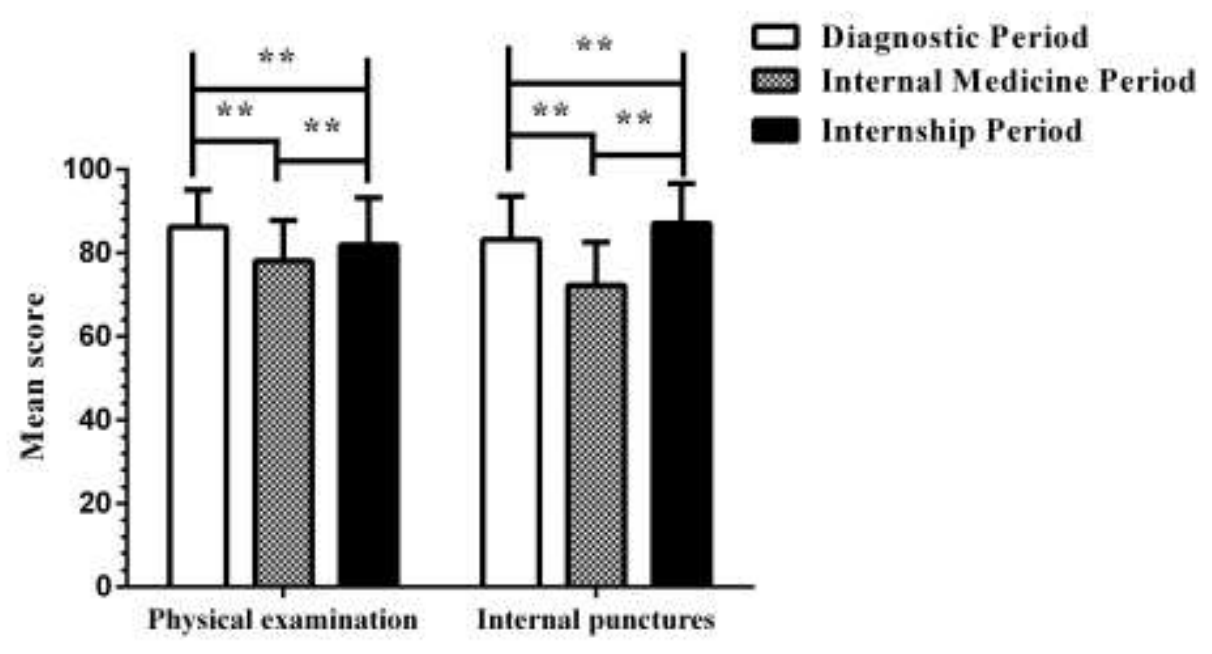

Figure 1. Scores on Physical Examinations and Internal Punctures Tests among the Diagnostic Period, Internal Medicine Period, Internship Period in the Class of 2010. **p<0.01 between two groups.

3.5 Comparison of the Mean Scores on Case Analysis Tests between the Internal Medicine Period and Internship Period in the Class of 2010, Illustrated in Table 4.

As illustrated in Table 4, P-values $0.492,>0.05$ were considered no statistically significant.

Table 4. Comparison of the Mean Scores of Case analysis between the internal medicine period and internship period in Grade 2010.

\begin{tabular}{lllcl}
\hline Indices & Internal Medicine Period $^{\#}$ & Internship Period $^{\#}$ & $\mathrm{t}$ & $P$ \\
\hline Case analysis & $76.91 \pm 8.86$ & $75.95 \pm 16.73$ & 0.69 & 0.492 \\
\hline
\end{tabular}

\#expressed as mean $\pm \mathrm{SD}$

\subsection{Questionnaire Survey}

3.6.1 In 'Feedback Opinion Form of Mastery of Clinical Basic Skills in Two Groups' (showed in Table 5), most students in observation group reflected that they could master blood and bone marrow examinations, ECG analysis, cardiovascular examination, cardio-pulmonary auscultation, neurological examinations, other examinations and theoretical knowledge well. Even 100\% students in this group considered that they were able to understand ECG analysis, cardiovascular auscultation and other system examination better; while lower percentage in control group were obtained. 
Table 5. Feedback Opinion Form of Mastery of Clinical Basic Skills in Two Groups

\begin{tabular}{|c|c|c|c|c|c|c|}
\hline \multirow[t]{2}{*}{ Indices } & \multicolumn{3}{|c|}{ Observation group ( $n=40)$} & \multicolumn{3}{|c|}{ Control group ( $n=37$ ) } \\
\hline & $\begin{array}{c}\text { Well } \\
\text { understood }\end{array}$ & $\begin{array}{l}\text { Moderately } \\
\text { understood }\end{array}$ & $\begin{array}{c}\text { Poorly } \\
\text { understood }\end{array}$ & $\begin{array}{c}\text { Well } \\
\text { understood }\end{array}$ & $\begin{array}{l}\text { Moderately } \\
\text { understood }\end{array}$ & $\begin{array}{c}\text { Poorly } \\
\text { understood }\end{array}$ \\
\hline Blood and bone marrow examinations & 25 & 13 & 2 & 15 & 20 & 2 \\
\hline ECG analysis & 30 & 10 & 0 & 17 & 17 & 3 \\
\hline Cardiovascular examination & 22 & 14 & 2 & 19 & 16 & 2 \\
\hline Neurological examination & 20 & 19 & 1 & 18 & 18 & 1 \\
\hline Other system examination & 29 & 10 & 1 & 22 & 14 & 1 \\
\hline Theoretical knowledge & 20 & 20 & 0 & 18 & 18 & 1 \\
\hline
\end{tabular}

3.6.2 Students' Satisfaction and Suggestions for the New Medical Teaching Mode

According to the Table 5, 39 students (98\%) in observation group were satisfied with interactive mode of medical or virtual simulation teaching and clinical practice; while 25 students $(66 \%)$ in control group were satisfied with the traditional teaching mode.

\subsection{A New Program Called System-oriented Curricula Was Initially Established}

In addition, one new program, called system-oriented curricula, was initially established, enrolling 16 outstanding students from the class of 2010 to promote the further mastery of clinical and basic knowledge. Multidisciplinary integration of clinical information and basic medical science, as well as clinical skills based on the cases in the ward or the scenarios, had been initially explored using case-based learning (CBL), problem-based learning (PBL), and team-based learning (TBL). In clinical practice, this integration can help the students to reinforce their comprehensive ability in theory with practice.

\subsection{Examples of System-oriented Curricula}

\subsubsection{Respiratory-system-based integrated Case Study}

First, a pleural effusion case was prepared, and teaching objectives and requirements were established. Students in this class were arranged into several groups to facilitate PBL discussion. Clinical information was gradually provided according to the students' clinical consideration. The tutors usually encouraged students to think and analyze diagnostic evidence in the case, which initiated brainstorming sessions combining basic theory and clinical knowledge until the primary diagnosis of pleural effusion was established. Additionally, the students were asked to review the basic medical science involved, including anatomy, pathology and physiology, microbiology, immunology, pharmacology and common symptoms, history taking, physical examination, laboratory investigations, medical images, etc. After discussion, the students were guided to the respiratory ward to learn and practice basic clinical skills such as history taking, physical examination, diagnostic process, and creating a treatment plan. A thoracentesis can be prescribed for this condition, and the students can conduct these techniques on the simulator until they become adept. Due to these methods, students achieved an overall understanding of the diagnosis and treatment of pleural effusion.

\subsubsection{Cardiovascular-system-based Integrated Case Study}

For this part, the students visited the ward. One case of acute chest pain was prepared to enable students to learn how to obtain the patient's medical history and perform a physical examination while at the same time considering the clinical diagnosis and treatment. Certain non-invasive techniques, such as ECG measurement and analysis, could be immediately taught to the students to identify diagnostic clues. Then, students were assigned case discussions in their groups. In this period, they could also review clinical thinking and basic knowledge including anatomy, histology and embryology, pathophysiology, pathology, etc. Once the diagnosis of acute myocardial infarction for this case became clear, the students were instructed to watch a percutaneous transluminal coronary angioplasty (PTCA) performed on the patient who had been observed in the first part. Consequently, vivid and real-life-scenario-based teaching was able to stimulate the students' interest in learning and greatly increase their impressions of the specific clinical situation. This approach also reflected an effective combination of CBL, PBL and TBL methods. 


\subsubsection{Digestive-system-based Integrated Case Study}

One acute abdominal pain case was prepared, and the teaching requirements and objectives were determined, including relevant knowledge of digestive anatomy, physiology, biochemistry, medical images, and diagnostic essentials of digestive common disorders. Then, the students were directed to the ward and endoscopy room in order to learn the clinical manifestations, diagnostics and therapeutic technology of digestive diseases. Subsequently, PBL discussion in each group, introduced by a real case, was held to analyze and confirm the most likely diagnosis and treatment plan. Most important for this system-oriented program was to allow the students to consider the clinical phenomena via real observation because they could follow the supervisor to watch the illness's progression.

\section{Discussion}

\subsection{Value of Constructing New Didactics in Internal Medicine Based on Translational Medicine and Patient Safety Concepts}

With integration between medical or virtual simulation teaching systems and hospital practice, clinical skills training can be improved. In this study, medical or virtual simulation instruments are linked to actual changes in behavior or clinical practice and in terms of patient safety or patient outcome(Alinier et al., 2014). New modes of instruction can provide medical students with more opportunities to enhance their clinical comprehensive ability and improve their professionalism. Therefore, this study is in accordance with translational medicine concepts and, simultaneously, considers patient safety. All alterations of the teaching approach sufficiently mobilized medical students' productive thinking and study interests and increased the interactivity and communication between teachers and students (Zhang, Cheng, Xu, Luo, \& Yang, 2015)

This research has developed an innovative didactic teaching approach combining medical or virtual simulation and clinical practice-based interactive teaching and learning in a diagnostics and internal medicine curriculum based on translational medicine and patient safety concepts. The study has emphasized the close integration between clinical theory and practice. The students have more opportunities to practice on the medical or virtual simulator after bedside teaching or contact with the real cases, which improves students' handling of the clinical issues because a large number of procedures, especially invasive techniques, cannot be performed on real patients by students. If any problems are found during the simulation practice, a new connection and cycle will be put into effect, and the students' efficiency can be evaluated in further clinical practice. As a comprehensive clinical skills training platform has been built at our hospital, new programs can be explored and designed to effectively train the students in the areas of professionalism, doctor-patient communication skills, clinical thinking and decision-making ability, disinfection concepts and techniques, adaptability and handling emergencies due to the combination of simulation teaching and real clinical practice.

\subsection{Comparison between Integrated Teaching Modes of Simulation Scenarios and Real Clinical Situations with Traditional Teaching Methods}

No statistically significant difference was observed between the observation group and control group in practical tests of PE, internal punctures and case analysis or in the theory-based examination of diagnostics and internal medicine. However, the questionnaire surveys showed that the observation group was more satisfied with the interactive teaching methods in medical or virtual simulation teaching and clinical practice than the control group was with the traditional teaching mode. Furthermore, because certain aspects of the teaching process can be harmful to the patient and should be considered, reasonable and effective integration of simulation and real-world clinical situations can achieve the same teaching efficiency and enhance the students' clinical thinking ability, doctor-patient communication skills, and clinical experience while ensuring patient safety. The present situation involves the continuous improvement of medical services for patients and increased awareness of self-protection. Many clinical procedures or operations are traumatic and even dangerous. If medical students whose clinical skills are not yet standardized conduct these procedures directly on patients, psychological and physical harm or even threats to the patients' lives can arise.

The traditional teaching program in class of 2009 students (control group) followed the approach proceeding from the classroom to the clinical training center to the hospital, lacking an effective combination of simulation and real cases. This approach could cause students' curt bedside manner when meeting patients at the early stages. For example, history taking and doctor-patient communication occur at the beginning of bedside teaching. The most difficult aspect for many medical students is to initiate a conversation with patients. They may feel embarrassed and nervous their first time encountering a patient in the ward. In clinical practice teaching, the students who examined the patient were relatively harsh in their attitude and the means they used to conduct examinations. They tended to 
palpate using stronger force on the patients' underlying organs such as liver, spleen, thyroid gland, etc. To address this issue, the present research studied and weighed the benefits and drawbacks with respect to patients' concerns and illness, students' practice, and teaching requirements. The new methods can both enhance the students' clinical comprehensive capacity and ensure patient safety.

4.3 The Changing Characteristics of Evaluation Scores among the Diagnostic Period, Internal Medicine Period and Internship Period in the Class of 2010 are in accordance with Teaching Objectives

The practical test scores on PE and internal punctures are relatively high because the evaluation in this period focused on standard learning approaches for the students. Students were expected to carefully master the key points and techniques of each skill, including patient safety, doctor-patient communication skills, aseptic concepts and methods, and correct and standard procedures. In contrast, the relatively low scores for the internal medicine period indicate that the students need to know more about clinical thinking, clinical applications, and decision-making ability in clinical cases, which present the most difficulty for them. Therefore, it is extremely important to integrate the simulation scenarios and real-world situations in the hospital during internal medicine study. The new teaching mode explored in this research played a key role in improving the students' comprehensive skills. As they accumulate more clinical experience in the internship rotation, the students can master PE and punctures more skillfully and precisely. The results will indicate whether traditional teaching and learning approaches should follow this change in approach. However, for case analysis tests, there was no statistically significant difference between the internal medicine period and the internship period. Case analysis requires comprehensive training and abilities including history taking, physical examination, diagnosis and treatment, and, in particular, clinical thinking, which is the area in which students have most difficulty increasing their scores in a short time and attaining mastery.

In view of this situation, the multidisciplinary integration of clinical information and basic medical science, as well as clinical skills based on cases in the ward or scenarios using case-based learning (CBL), problem-based learning (PBL), and team-based learning (TBL) methods in clinical practice, can help students learn to consider and solve clinical problems. The respiratory, cardiovascular, and urinary systems and their common disorders were introduced, and students were guided to discuss and practice to increase their clinical thinking abilities. The effectiveness and implementation of this approach should be further analyzed and improved(Lu, Huang, Li, Feng, \& Yang, 2017).

\section{Conclusion}

This research, with its high integration among simulation scenarios, relevant practices and actual clinical problems based on translational medicine concepts (McGaghie, Issenberg, Barsuk, \& Wayne, 2014), can greatly shorten the distance from the laboratory to the hospital and ensure patient safety, thereby enhancing students' clinical comprehensive ability and ultimately achieving satisfactory teaching effects. Moreover, novel modes of instruction are more popular with the students. In addition, in each period of clinical education, the characteristics of clinical skills teaching and learning may be different, and teachers should continue to explore reasonable pedagogical approaches to develop system-oriented curricula with diverse teaching methods.

\section{Limitations and Future Directions}

In the future research, we should pay more attention to the comprehensive cultivation of clinical thinking and ability. Especially, for case analysis training, it needs the combination between theory and clinical practice based on the scenarios and real cases in the medical simulation center or in the teaching hospital. More teaching methods and accurate design will be required and explored and in clinical education.

\section{Acknowledgments}

The authors would like to thank the large number of academic staff and medical students who contributed to the research and the usual teaching in first clinical medicine college and medical college of Jinan University. The authors appreciated the help of Dr. Yanghai Zhou from the first affiliated hospital of Jinan University and Professor Renbin Qi from the department of pathophysiology, school of basic medicine, Jinan University.

\section{Compliance with Ethical Standards:}

Declaration of Interest: The authors have no conflicts of interest to report.

Ethics and consent: The research was conducted in accordance with the Helsinki Declaration of 1975, as revised in 2008. The medical student participants were informed in written and oral remarks. During the whole process, the patients' privacy had to be considered and protected, and no invasive and harmful procedures were performed on them. In addition, the data of the study were accessible to the authors. 


\section{References}

Alinier, G., Harwood, C., Harwood, P., Montague, S., Huish, E., Ruparelia, K., \& Antuofermo, M. (2014). Immersive clinical simulation in undergraduate health care interprofessional education: Knowledge and perceptions. Clinical Simulation in Nursing, 10(4), e205-e216. https://doi.org/10.1016/j.ecns.2013.12.006

Back, D. A., Haberstroh, N., Antolic, A., Sostmann, K., Schmidmaier, G., \& Hoff, E. (2014). Blended learning approach improves teaching in a problem-based learning environment in orthopedics - a pilot study. BMC Med Educ, 14, 17. https://doi.org/10.1186/1472-6920-14-17

Bond, W., Kuhn, G., Binstadt, E., Quirk, M., Wu, T., Tews, M., . . Ericsson, K. A. (2008). The Use of Simulation in the Development of Individual Cognitive Expertise in Emergency Medicine. Academic Emergency Medicine, 15(11), 1037-1045. https://doi.org/10.1111/j.1553-2712.2008.00229.x

Bond, W. F., Lammers, R. L., Spillane, L. L., Smith-Coggins, R., Fernandez, R., Reznek, M. A., . . Gordon, J. A. (2007). The use of simulation in emergency medicine: A research agenda. Academic Emergency Medicine, 14(4), 353-363. https://doi.org/10.1197/j.aem.2006.11.021

Cohrs, R. J., Martin, T., Ghahramani, P., Bidaut, L., \& J, P. (2015). Translational Medicine definition by the European Society for Translational Medicine. New Horizons in Translational Medicine 3(2), 86-88. https://doi.org/10.1016/j.nhtm.2014.12.002

Dai, K.-R., Yang, F., \& Gan., Y.-K. (2013). Development of translational medicine in China: Foam or feast? Journal of Orthopaedic Translation, 1, 6-10. https://doi.org/10.1016/j.jot.2013.07.003

Gaba, D. M. (2007). The future vision of simulation in healthcare. Simul Healthc, 2(2), 126-135. https://doi.org/10.1097/01.SIH.0000258411.38212.32

Lu, C., Huang, S., Li, Z., Feng, L., \& Yang, J. (2017). Effects of Exploratory and Heuristic Multi-methods on System-oriented Curricula Based on Clinical Scenarios. International Journal of Higher Education, 6(6), 55. https://doi.org/10.5430/ijhe.v6n6p55

McGaghie, W. C., Issenberg, S. B., Barsuk, J. H., \& Wayne, D. B. (2014). A critical review of simulation-based mastery learning with translational outcomes. Med Educ, 48(4), 375-385. https://doi.org/10.1111/medu.12391

Ming-ming, Z., Yu-rong, D., \& Jing, L. (2010). Patient Safety Education: One of the Important Topics for 21st Medical Education. Chinese Journal of Evidence-based Medicine, 10(6), 637-639.

Miyasaka, K. W., Buchholz, J., LaMarra, D., Karakousis, G. C., \& Aggarwal, R. (2015). Development and implementation of a clinical pathway approach to simulation-based training for foregut surgery. $J$ Surg Educ, 72(4), 625-635. https://doi.org/10.1016/j.jsurg.2015.01.017

Moral, I. d., \& Maestre., J. M. (2013). A view on the practical application of simulation in professional education. Trends in Anaesthesia and Critical Care, 3, 146-151. https://doi.org/10.1016/j.tacc.2013.03.007

Vattanavanit, V., Kawla-Ied, J., \& Bhurayanontachai, R. (2017). High-fidelity medical simulation training improves medical students' knowledge and confidence levels in septic shock resuscitation. Open Access Emerg Med, 9, 1-7. https://doi.org/10.2147/OAEM.S122525

Woolf, S. H. (2008). The meaning of translational research and why it matters. JAMA, 299(2), 211-213. https://doi.org/10.1001/jama.2007.26

Xi-ming, D., \& Jun-hon, F. (2014). Thinking on medical postgraduate education bade on development of translational medicine. Journal of Qiqihar University of Medicine, 35(4), 564-565.

Yuan, G. Y. (2014). A preliminary study of applying translational medicine in clinical training for clinical medical students. China Higher Medical Education, 11, 79-80.

Zhang, M. Y., Cheng, X., Xu, A. D., Luo, L. P., \& Yang, X. (2015). Clinical simulation training improves the clinical performance of Chinese medical students. Med Educ Online, 20, 28796. https://doi.org/10.3402/meo.v20.28796 